\title{
Segmental Pigmentation Disorder with Congenital Heterochromia Iridis
}

\author{
Carmen Madrigal Díez ${ }^{*}$, Sara Rodríguez Prado², José Héctor Fernández Llaca ${ }^{3}$ \\ ${ }^{1}$ Primary Paediatric Health Care, Centro de Salud Bezana, Servicio Cántabro de Salud, Spain \\ ${ }^{2}$ Department of Opthalmology, Hospital de Sierrallana, Torrelavega, Cantabria, Spain \\ ${ }^{3}$ Department of Dermatology, Hospital Universitario Marqués de Valdecilla, Santander, Cantabria, Spain \\ Email: ${ }^{*}$ c.madrigaldiez@yahoo.es
}

Received 15 June 2015; accepted 25 August 2015; published 28 August 2015

Copyright (C) 2015 by authors and Scientific Research Publishing Inc.

This work is licensed under the Creative Commons Attribution International License (CC BY).

http://creativecommons.org/licenses/by/4.0/

(c) $\underset{\mathrm{EY}}{\mathrm{O}}$ Open Access

\begin{abstract}
We report the case of a 10-year-old girl with congenital complete heterochromia iridis and segmental pigmentation disorder in its hyperpigmented form. We have found no publication that mentions the combination of these 2 disorders.
\end{abstract}

\section{Keywords}

Congenital Heterochromia Iridis, Segmental Pigmentation Disorder, Café-au-Lait Macules

\section{Case Report}

During a routine checkup, a 10-year-old girl had noticeably different colors in her irises and had large hyperpigmented spots on her skin. According to her mother, both irises were blue during the child's first month of life; however, in the following months, the left iris remained blue while the right darkened until reaching its definitive brown color by 10 months of age. During her second year of life, two spots appeared on the child's back that were darker brown than her normal skin, which progressively spread towards the front. During the summer when the child was 10 years of age, her family noticed a spot on the left side of her face with a similar color to that on her chest. This new spot had not been detected before. During the autumn, the spot progressively faded until it almost disappeared. The girl's development has been normal, with no clinical suspicion of endocrine, sensory or neurological disorders or other significant manifestations. There is no similar medical history in the girl's family.

The physical examination confirmed the presence of two large café-au-lait spots, with irregular coloration and edges. The spots extended from the lumbosacral region towards both sides of the trunk through the flanks

\footnotetext{
${ }^{*}$ Corresponding author.
}

How to cite this paper: Madrigal Díez, C., Rodríguez Prado, S. and Fernández Llaca, J.H. (2015) Segmental Pigmentation Disorder with Congenital Heterochromia Iridis. Open Journal of Pediatrics, 5, 213-217. 
(Figure 1) until reaching the spot on the right side of the midline of the abdomen, where it ended neatly and without going past the line (Figure 2). The lateral edges of both spots were more irregular. In the paravertebral region, the intensity of the pigmentation progressively diminished until it was almost imperceptible. In the right intercostal area of the chest, the girl had another isolated spot of the same color as the previous ones although smaller. On the left side of the face, there was another hyperpigmented spot, with an irregular border that extended over the forehead, the periorbital region and the cheek. On the forehead, the border between the normal skin and the hyperpigmented skin was well defined and showed a tendency to respect the midline, although it slightly exceeded it in the upper area (Figure 3). In the periorbital area and cheek, the borderline was diffuse, and the pigmentation faded until disappearing (Figure 4). We also observed complete heterochromia iridis, with the right iris going brown and the left remaining blue (Figure 3). A thorough ophthalmologic examination ruled out the presence of any underlying refractive problem or ocular disease. The girl's physical and psychological development was normal, and there were no other associated organ disorders or signs of advanced puberty.

The results of an extensive laboratory study were normal and included serum calcium and phosphate levels (10.02 and $4.19 \mathrm{mg} / \mathrm{dl}$, respectively) and 24-hour phosphaturia (950 ml daily and $864 \mathrm{mg} / \mathrm{dl}$ ). The hormone levels of prolactin, TS4, T4, LH, FSH and estradiol were also normal for a prepubescent girl. There were no apparent skeletal disorders (including the head) in the radiological examination. The patient's bone age (10 years) matched her chronological age. The girl and her family rejected the possibility of performing skin biopsies.

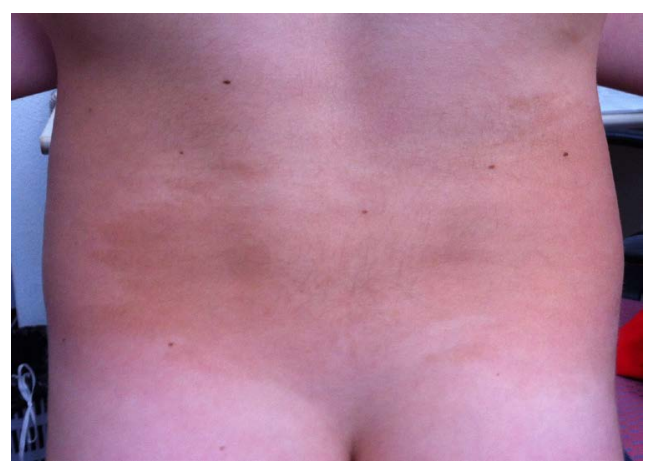

Figure 1. Two lumbosacral bilateral café-au-lait macules, with irregular edges. Their color fades out as they approach the dorsal midline.

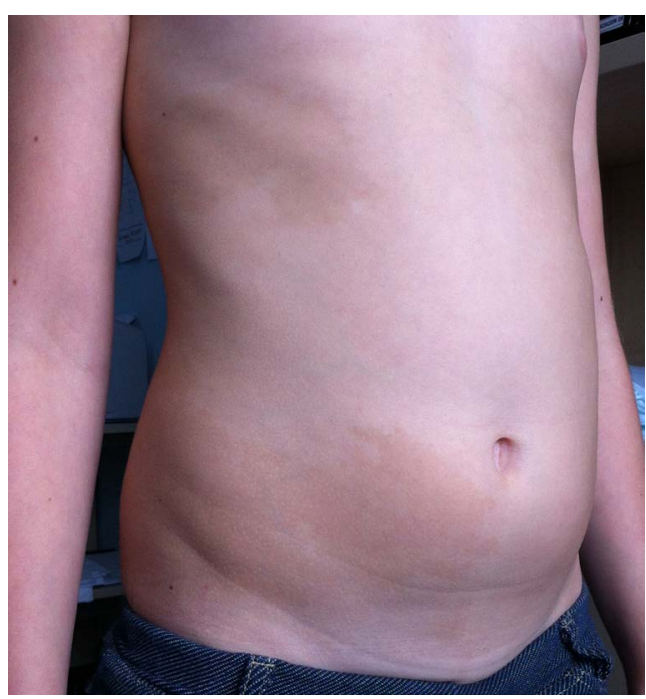

Figure 2. On the right side, the macule stops with a clean edge at the ventral midline without going over it. In the intercostal region, another less extensive macule can be observed. 


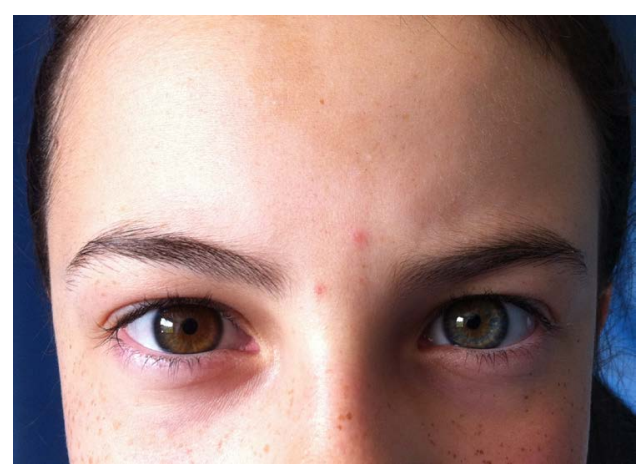

Figure 3. Café-au-lait facial macule. In the front left region, the macule slightly surpasses the midline; its edge is clearly delimited from the unaffected skin. Complete heterochromia iridis, with a darker right iris.

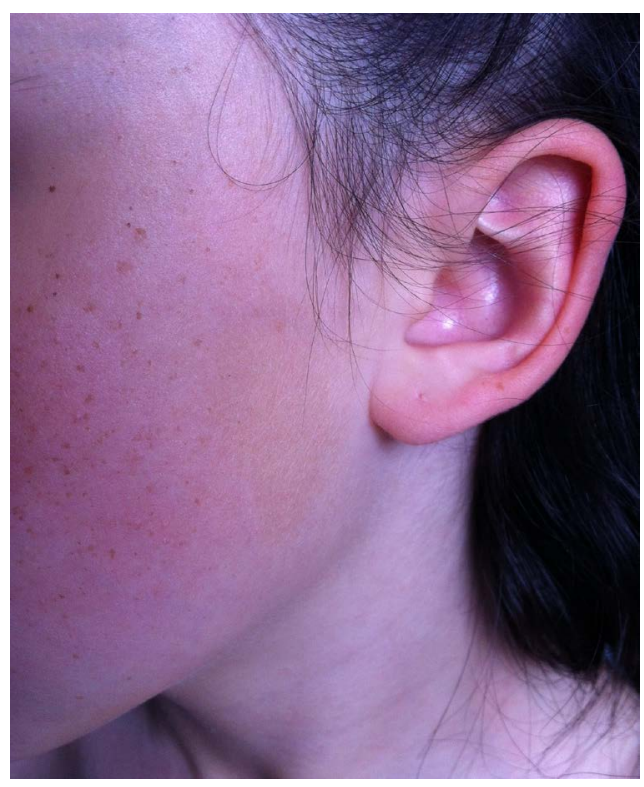

Figure 4. On the cheek, the edge is more blurred.

\section{Discussion}

Eye and skin color are two of the most distinctive personal appearance traits. The two have a common process that creates them: the migration of melanoblasts from the neural crest to their destination points, their maturation to melanocytes and the metabolic process of formation and distribution of melanin. However, while skin melanocytes release melanosomes to the surrounding cells, the uveal melanocytes do not release them [1].

Complete congenital heterochromia iridis (CCHI) and the café-au-lait macules (CALM) are two pigmentation disorders of the eyes and skin. At birth, neither the migration or maturation processes of the iris melanocytes have been completed. Therefore, CCHI is not typically observed until after the first months of life [1]. The same occurs with CALM, which can go unnoticed for the first few years, especially in children with pale skin [2].

CCHI can present as an isolated disorder or associated with other organ disorders. With an incidence lower than 1/200.000, isolated CCHI is included by the Genetic and Rare Diseases Information Center (GARD) within the rare diseases group. Other times, CCHI is included in diseases or syndromes that, along with other symptoms, include heterochromia iridis due to hypochromia (Hirschsprung's disease, congenital Horner's syndrome, Waardenburg syndrome and hypomelanosis of Ito) or to hyperchromia of the affected iris (oculodermal melanocytosis or nevus of Ota (ODM) and Sturge-Weber syndrome). 
Our patient is a carrier of complete heterochromia iridis, which, as occurs with CCHI, was already apparent in the first months of life, with no apparent associated sign that would lead to the suspicion that the patient had Hirschsprung's disease, Horner's syndrome, Waardenburg syndrome, Sturge-Weber syndrome or hypomelanosis of Ito.

In our patient, the simultaneous onset of CCHI with diffuse macular edges, observed at prepubescence in skin areas innervated by the first two branches of the trigeminal nerve, with diffuse edges and with seasonal variations in intensity, could suggest an ODM. However, the skin macula was soft brown and on the left side of the face, while the brown iris was in the right eye. In contrast, facial macula in ODM tends to be grey-blue, and the dark iris is on the same side as the skin macula [2] [3].

In CALM, there is an increase in the melanin contained in the basal melanocytes and keratinocytes, with no increase in the quantity of melanocytes [3] [4]. CALM are more common than CCHI, especially when they are few, small, isolated, symmetrical and well-defined, which occurs in more than $10 \%$ of healthy children. In such cases, CALM represents a personal characteristic, with no pathological meaning. CALM are significantly rarer when they increase in number and size, with asymmetric forms and irregular edges [2] [4].

The characteristics of our patient's macules are similar to those of segmental pigmentation disorder (SegPD), a condition described by Metzker [5] and in subsequent reviews by Hogeling [6]. The name was employed to refer to a subgroup of patients with large hypopigmented or hyperpigmented skin macules, which had well-defined borders in the body's midline, especially the ventral. These macules do not usually spread beyond the midline, although they occasionally overlap by a few centimeters [6], and have more diffuse lateral edges. Of the patterns described by Happle [7] as a reflection of mosaic skin pigmentation, the archetype that most resembles the form and distribution of our patient's macules is type 2 checkerboard. However, it is not always possible to fit all SegPDs in this classification [8], which likely would extend with the incorporation of an additional archetype [9]. The histological findings in cases of SegPD with hyperpigmentation in which skin biopsies were performed have been similar to those found with classic CALM [4] [5]. Therefore, the current criteria for the diagnosis of SegPD are mainly clinical [6] [8] [10]. The risk that SegPD is associated with other systemic disorders is low; however, in cases with highly extensive spots, a more thorough study that rules out their presence is recommended [6] [10].

In the forms with hyperpigmentation, the differential diagnosis should be established with McCune-Albright syndrome (MAS), giant and isolated CALMs, neurofibromatosis (NF), speckled lentiginous nevus and nevoid hyperpigmentation [2] [9].

Although they do not always match these characteristics, CALMs of MAS are typically described as large, unilateral macules, with irregular and asymmetrical edges ("coast of Maine” pattern), not exceeding the midline, following the broad band pattern of Blaschko's lines and are accentuated with exposure to the sun [2] [11]. These macules are usually the first observed manifestation of MAS. They should therefore not be underestimated, and their observation should lead to the necessary tests to rule out the presence of fibrous dysplasia and/ or undetected hyperfunctioning endocrinopathies, which, when present, are detectable before 10 years of age in the vast majority of cases [9] [11]. For our patient, the normal results from the endocrine and radiological tests allowed us to reasonably rule out MAS.

The large isolated CALMs preserve the morphological peculiarities of classic CALMs, without the lateral blurring seen with our patient and with no relation to the midline [4] [6].

CALMs of NF usually present the "coast of California” pattern; their abundance in the axilla and groin originate the Crowe sign. The neurofibromas usually appear later, during adolescence. Lisch nodules, which can change the color of the iris, are present in both eyes, usually appear during late childhood and are more abundant in the lower hemisphere [1]. Segmental NF is a variant of NF caused by a postzygotic mosaicism and only presents in a body segment in which the characteristic manifestations of NF appear [1] [8]. For our patient, the CALMs did not correspond to the coast of California pattern. The heterochromia was present at birth, and she did not have Crowe's sign or Lisch nodules.

Speckled lentiginous nevus can present demarcation in the body midline, but the characteristic darker specks that stand out over the flat brown macula are very obvious at 10 years of age [6].

Nevoid hyperpigmentation follows the pattern of narrow Blaschko lines, which is very different from that of our case.

Although not a rare clinical condition, the SegPD described by Metzker is still not well defined. This lack of definition is due in part to the considerable diversity in terminology with which the condition is reported and to 
the lack of clarity in terms of its clinical characteristics and potential associations, which makes it more difficult to study and characterize [6] [8]. Therefore, it is advisable to report the characteristics of SegPD observed during clinical practice [8].

We have presented a case of a girl with SegPD, who was also a carrier of CCHI but with no other associated anomaly. Both CCHI and SegPD are the results of a disorder in the biological process responsible for melanin pigmentation in the iris and skin, respectively. Although skin pigmentation disorders associated with ocular disorders have been reported, we have found no reports that refer to the simultaneous onset of SegPD and CCHI in one individual. There has been a reported case of an 11-year-old girl with a large facial CALM associated with CCHI, who also had a large hemangioma in one of the buttocks [12]. The report does not explicitly reference the possibility of the condition being a SegPD, and the skin lesion was considered a large segmental CALM.

Due to its incidence, which is estimated at approximately $0.35 \%$ [6] [7], SegPD cannot be considered a rare disease; however, the very low incidence of CCHI means that the probability of the two conditions casually coinciding is very low $\left(<1 / 57 \times 10^{6}\right)$. Reports of other cases in which the two disorders coincided would suggest some type of correlation between them.

\section{Conflict of Interest}

The authors declare no conflict of interest.

\section{References}

[1] Rennie, I.G. (2012) Don’t It Make My Blue Eyes Brown: Heterochromia and Other Abnormalities of the Iris. Eye, 26, 29-50. http://dx.doi.org/10.1038/eye.2011.228

[2] Shah, K.N. (2010) The Diagnostic and Clinical Significance of Café-au-Lait Macules. Pediatric Clinics of North America, 57, 1131-1153. http://dx.doi.org/10.1016/j.pcl.2010.07.002

[3] Sinha, S., Cohen, P.J. and Schwartz (2008) Nevus of Ota in Children. Cutis, 82, 25-29.

[4] Landau, M. and Krafchik, B.R. (1999) The Diagnostic Value of Café-au-Lait Macules. Journal of the American Academy of Dermatology, 40, 877-890. http://dx.doi.org/10.1016/S0190-9622(99)70075-7

[5] Metzker, A., Morag, C. and Weitz, R. (1983) Segmental Pigmentation Disorder. Acta Dermato-Venereologica, 63, 167-169.

[6] Hogelin, M. and Frieden, I.J. (2010) Segmental Pigmentation Disorder. British Journal of Dermatology, 162, 13371341. http://dx.doi.org/10.1111/j.1365-2133.2010.09702.x

[7] Happle, R. (1993) Mosaicism Human Skin Understanding the Patterns and Mechanism. JAMA Dermatology, 129, 1460-1470. http://dx.doi.org/10.1001/archderm.1993.01680320094012

[8] Orion, E., Matz, H. and Wolf, R. (2003) Café au lait Has a Hue of Its Own. Dermatology Online Journal, 9, 8.

[9] Happle, R. and Bittar, M. (2006) Patrones del mosaicismo en la piel humana: Comprendiendo aspectos actuales y futuros. Dermatol Pediatr Lat, 4, 171-181.

[10] Treta, J. (2010) Patterned Pigmentation in Children. Pediatric Clinics of North America, 57, 1121-1129. http://dx.doi.org/10.1016/j.pcl.2010.07.007

[11] Collins, M.T., Cantante, R.F. and Eugster, E. (2012) McCune-Albright Syndrome and the Extraskeletal Manifestations of Fibrous Dysplasia. Orphanet Journal of Rare Diseases, 7, S4. http://www.ojrd.com/content/7/S1/S4

[12] Quilan, K. and Shwayder, T. (2005) Café au lait Macule Associated with Heterochromia Iridis. Pediatric Dermatology, 22, 177-178. http://dx.doi.org/10.1111/j.1525-1470.2005.22220.x 